\title{
Epidemiologia e impacto social
}

Maurício Silva de Lima ${ }^{1}$

\section{Resumo}

A formulação de políticas em saúde mental depende essencialmente de informações a respeito da frequiência e distribuição dos transtornos depressivos. Nos últimos 15 anos, pesquisas de base populacional em epidemiologia psiquiátrica têm sido conduzidas, gerando conhecimento detalhado sobre a freqüência, fatores de risco, incapacidade social, e uso de serviços de saúde. Neste artigo, dados sobre a epidemiologia da depressão são discutidos, a partir de resultados de recentes pesquisas populacionais: o estudo da Área de Captação Epidemiológica do Instituto Nacional de Saúde Mental dos Estados Unidos (ECA- NIMH), a Pesquisa Nacional de Co-morbidade (NCS), a pesquisa de Morbidade Psiquiátrica na Grã-Bretanha (OPCS), o Estudo Brasileiro Multicêntrico de Morbidade Psiquiátrica, e outras pesquisas conduzidas no Brasil em atenção primária. As prevalências de depressão maior e de distimia, bem como a de outros transtornos depressivos, são altas, independente do lugar onde a pesquisa foi conduzida, tipo de instrumento diagnóstico usado, e dos períodos de tempo para os quais a prevalência se aplica. Depressão é mais comum entre mulheres, pessoas divorciadas ou separadas, vivendo sozinhas, com baixo nível de escolaridade e renda, desempregados e morando em zonas urbanas. Pessoas deprimidas são mais sujeitas a consultarem médicos e a serem hospitalizadas. O custo e a eficácia dos tratamentos para depressão devem ser balanceados com o alto custo individual e social associados à enfermidade.

\section{Descritores}

Depressão; transtorno depressivo; prevalência; incidência; impacto social

\section{Abstract}

Information on the epidemiology of depressive disorders is essential for providing a framework for the formulation of effective mental health policies. In the last 15 years, some population surveys of psychiatric morbidity in adults have been conducted and, as a result, details on the frequency, risk factor, social disabilities, and service use are now available. Epidemiological findings for depressive illness are discussed on the light of results from recent mass surveys namely the Epidemiological Catchment Area Study (ECA), the National Comorbidity Survey (NCS), the OPCS Survey of Psychiatric Morbidity in Great Britain, the Brazilian Multicentric Study of Psychiatric Morbidity, and other surveys on common mental disorders conducted in Brazil. Prevalence rates for major depression disorder, dysthymia, and other depressive states are high for all estimates, regardless setting, the type of instrument used for generating psychiatric diagnosis, and the time periods by which prevalence is defined. Depression is more common among women, divorced or separated people, those living in one-person family units, with lower level of education and income, unemployed and urban dwellers. Depressed subjects are more likely to have medical consultations and in-patient episodes. The cost-effectiveness of treatments must be balanced with the high individual and social impact associated to depressive illness.

\section{Keywords}

Depression; depressive disorders; prevalence; incidence; social impact

\section{Introdução}

Estudos epidemiológicos conduzidos nas últimas duas décadas têm proporcionado uma compreensão mais ampla da ocorrência e do curso dos transtornos mentais, além de permitir que se avaliem consequiências diretas e indiretas das doenças, como prejuízo no funcionamento individual, familiar e social. Essas informações servem como uma base para decisões políticas em saúde mental, bem como para a avaliação do acesso à assistência médica e uso de serviços de saúde. ${ }^{1}$

O conhecimento da epidemiologia dos transtornos do humor complementa a investigação clínica, conferindo ao quadro clínico amplitude e uma dimensão temporal. ${ }^{2}$ Tal informação é essencial para a adequada compreensão da história natural da depressão, uma vez que os clínicos convivem com pacientes deprimidos, principalmente quando da exacerbação sintomática de transtornos crônicos, ou em quadros agudos. Além disso, estudos prospectivos e de seguimento - as coortes - fornecem informações sobre outros aspectos das doenças depressivas, tais como fatores de risco e prognósticos, além de tendências históricas.

A maioria dos dados a serem apresentados nesta revisão são 
relativos à depressão maior e à distimia, transtornos depressivos mais graves e/ou persistentes. As informações são mais limitadas no que diz respeito à distribuição e fatores associados a transtornos depressivos leves ou moderados e de curta duração. Tais transtornos, no entanto, são igualmente importantes em função de sua alta prevalência na comunidade e em serviços de atenção primária à saúde.

\section{Estudos de identificação de caso}

Casos identificados a partir de critérios diagnósticos padronizados, como aqueles daDSM-IV ${ }^{3}$ e CID $10,{ }^{4}$ representam uma mistura heterogênea que pode ter pouca relevância clínica. O limite entre depressão clínica e flutuações normais no humor não é claro mesmo a presença ou ausência de sintomas pode ser controversa. Indivíduos na comunidade podem apresentar síndromes depressivas que não preencham os critérios diagnósticos acima citados, mas nem por isso os problemas deixam de ser incapacitantes. Doenças mentais comuns, ou transtornos psiquiátricos menores, são condições 'maiores' quando se considera que causam enorme sofrimento, se associados com incapacitação e alto custo individual, social e econômico. ${ }^{5}$

O processo de identificação de casos nas pesquisas populacionais mais recentes foi feito através de instrumentos diagnósticos desenvolvidos em hospitais e ambulatórios, sendo por isso mais acurados para pacientes com síndromes mais severas. De forma similar, o diagnóstico de co-morbidade nessas investigações costuma ser impreciso. É difícil as pessoas lembrarem o que surgiu primeiro, por exemplo, a depressão ou a ansiedade. Na prática clínica, freqüentemente elas coexistem.

Considerando-se tanto as vantagens quanto as dificuldades e limitações inerentes às pesquisas de base populacional, procurase neste artigo limitar a discussão a estudos recentes de grandes amostras, cujas características metodológicas essenciais são descritas abaixo. Dados do estudo multicêntrico brasileiro e de outras pesquisas locais serão também apresentados, dada a sua relevância para planejamento local em saúde. A comparação de diferentes populações, em estudos de base populacional de boa qualidade e que utilizaram métodos semelhantes, pode permitir uma avaliação mais abrangente do conhecimento da distribuição da depressão na população geral.

\section{Descrição dos estudos incluídos nesta revisão}

- O Estudo da Área de Captação Epidemiológica do Instituto Nacional de Saúde Mental (ECA- NIMH), ${ }^{1}$ nos Estados Unidos, foi a primeira grande pesquisa epidemiológica, de base populacional, que utilizou critérios diagnósticos padronizados para a definição de transtornos psiquiátricos (DIS - Diagnostic Interview Schedule). O ECA foi conduzido em cinco centros (New Heaven, Eastern Baltimore, St. Louis, Durham e Los Angeles), com uma amostra total de aproximadamente 20 mil pessoas. $O$ índice de perdas nesse estudo variou de $20 \%$ a $25 \%$, e seus achados indicam que cerca de $1 / 4$ das pessoas, em algum momento da sua vida, apresentam algum transtorno de ansiedade de acordo com os critérios do DSM-III.

- A Pesquisa Nacional de Co-morbidade (NCS), nos Estados Unidos, ${ }^{6}$ foi realizada após o ECA, utilizando critérios diagnósti- cos baseados no DSM-III-R. Enquanto o ECA foi delineado para ser um estudo de prevalência, esta pesquisa objetivava avaliar também fatores de risco. Os diagnósticos foram gerados a partir de uma versão modificada do CIDI (Composite International Diagnostic Interview), que por sua vez é baseada no DIS. Foram entrevistadas 8.098 pessoas, com idades variando entre 15 e 54 anos. A taxa de respondentes foi de $82,4 \%$. Esse estudo mostrou que a maioria das pessoas $(56 \%)$ com transtornos mentais apresentava co-morbidade com outras condições psiquiátricas.

- A Pesquisa Nacional de Morbidade Psiquiátrica na GrãBretanha $^{7,8}$ (OPCS) foi planejada para estudar a prevalência de transtornos psiquiátricos, bem como a extensão da incapacidade associada, co-morbidade e utilização de uso de serviços de saúde. Além disso, o objetivo também foi investigar fatores precipitantes das doenças e suas associações com diferentes estilos de vida. A população-alvo constituiu-se de adultos com idades entre 16 e 64 anos, vivendo na Inglaterra, País de Gales e Escócia. Quatro amostras separadas foram entrevistadas: domicílios privados, uma amostra suplementar com pessoas sofrendo de psicose e vivendo em suas residências, instituições para doenças mentais e um quarto grupo incluindo pessoas sem domicílio fixo, vivendo na rua ou em instituições próprias para indivíduos sem moradia. Os diagnósticos foram gerados a partir de entrevistas utilizando o CIS-R (Clinical Interview Schedule, Revised).

- O Estudo Multicêntrico Brasileiro de Morbidade Psiquiátri$c a^{9}$ obteve estimativas de prevalência de transtornos mentais em três grandes centros urbanos brasileiros: Brasília, São Paulo e Porto Alegre, utilizando os critérios diagnósticos do Manual Diagnóstico e Estatístico da Associação Psiquiátrica Americana (DSM-III). Na primeira fase, foi utilizado o Questionário sobre Morbidade Psiquiátrica em Adultos (QMPA); na segunda fase, as pessoas com escore acima de 7 no QMPA foram reentrevistadas, desta vez por uma equipe de psiquiatras especialmente treinada, que utilizou a lista de checagem de sintomas do DSM-III.

\section{Freqüência dos transtornos depressivos Incidência}

A incidência dos transtornos de humor representa o número de novos casos de doença que surgem em uma população em risco de adoecer (ou seja, pessoas que não apresentam a doença no início do estudo), em um determinado período de tempo. Os estudos de coorte ou de seguimento fornecem estimativas de incidência, mas são dispendiosos e executados a longo prazo. Por isso, os dados de incidência sobre transtornos mentais em geral são escassos.

Uma vez que depressão maior é comum e tende à remissão e recorrência, a incidência é relativamente alta. Blazer ${ }^{2}$ afirma que a incidência anual de depressão maior encontrada no estudo ECA foi de $1,59 \%$.

Uma pesquisa em Lundby (Suécia) ${ }^{10}$ mostrou uma incidência anual de depressão de $0,43 \%$ em homens e $0,76 \%$ em mulheres. Até a idade de 70 anos, a probabilidade acumulativa de um primeiro episódio de depressão foi de $27 \%$ para homens e $45 \%$ para mulheres, taxas que colocam a depressão como um dos mais importantes problemas de saúde pública. 


\section{Prevalência}

A tabela 1 apresenta os resultados das principais investigações epidemiológicas. Ela sugere que podem existir importantes variações na freqüência de transtornos depressivos, o que torna a comparação entre os achados uma tarefa complexa. As diferenças encontradas são provavelmente devidas aos delineamentos utilizados nas pesquisas. A utilização de diferentes critérios diagnósticos a partir de diferentes instrumentos, bem como os diversos períodos de tempo para os quais a prevalência se aplica, determinam que as medidas de efeito resultantes sejam de difícil síntese. É possível ver, no entanto, que tanto a depressão maior como a distimia e outros transtornos depressivos de curta duração são doenças comuns, que afetam um grande número de pessoas em diferentes países e culturas.

No caso do estudo brasileiro, as estimativas de prevalências foram geradas a partir de uma subamostra com um número reduzido (836). A ponderação para a grande amostra (6.476) acarreta diminuição da precisão e gera prevalências para grupos diagnósticos, e não para transtornos individuais.

Ainda de acordo com o estudo ECA, a idade média de começo da depressão maior é em torno de 27 anos. A duração média do transtorno seria de 9 anos. Esses achados são consistentes com outras investigações, que colocam a depressão como uma doença de caráter crônico e de início na terceira década de vida.

Outras pesquisas populacionais e em atenção primária à saúde foram conduzidas no Brasil, investigando principalmente a ocorrência dos chamados 'transtornos psiquiátricos menores', que compreenderiam principalmente os quadros neuróticos de depressão, ansiedade e somatoformes. Iacoponi (1989) encontrou uma prevalência desses transtornos em cerca de metade dos pacientes em serviços de atenção primária à saúde em São Paulo. Na população geral, estudos conduzidos em São Pau$10^{11}$ e Pelotas ${ }^{12}$ produziram estimativas muito semelhantes com relação aos transtornos psiquiátricos menores: $12 \%$ entre os homens e $25 \%$ entre as mulheres no primeiro estudo e $18 \%$ e $27 \%$, respectivamente, no segundo.

\section{Fatores de risco}

Os fatores demográficos que têm sido associados à depressão, em pesquisas populacionais, são relativos a sexo, idade e raça. Os dois primeiros têm sido replicados em diferentes con- textos, enquanto que os achados relativos à raça são variados e podem ser determinados por fatores de confusão, tais como renda e escolaridade.

Com relação ao sexo, todas as pesquisas citadas acima indicam que as mulheres apresentam cerca de duas vezes mais depressão do que homens. No entanto, o sexo feminino parece não ser um fator de risco 'per se', mas sim o ambiente e suporte social na maioria das culturas. $\mathrm{O}$ fato de os homens relatarem menos sintomas depressivos do que as mulheres também colabora para esse achado.

A idade de começo dos transtornos depressivos situa-se entre 20 e 40 anos, apesar de a depressão também ocorrer na infância. De forma similar ao sexo, também não há porque pensar que a idade seja um fator de risco isoladamente. Fatores sociais podem colocar as pessoas mais jovens em maior risco, da mesma forma que a predisposição biológica para depressão maior pode aumentar com a idade ${ }^{2}$. Estudos brasileiros conduzidos na comunidade sugerem que transtornos mentais comuns, como ansiedade, depressão e transtornos somatoformes, aumentam com a idade. ${ }^{11,12}$

Com relação à raça, no ECA não foram encontradas diferenças. Tais diferenças poderiam ser explicadas por fatores sócioeconômicos, sendo o ECA o primeiro estudo que permitiu comparações diretas entre brancos, afro-americanos e hispânicos. As diferenças eventualmente encontradas desapareceram quando, na análise, foram controlados fatores como idade, local de residência e situação sócioeconômica.

Fatores sócioeconômicos: vários estudos populacionais sugerem que pessoas com baixa escolaridade e renda apresentam maiores prevalência de transtornos mentais. No ECA, com exceção dos resultados encontrados na Carolina do Norte, a associação entre baixa renda e depressão foi fraca. No estudo americano de co-morbidade, as pessoas com menor renda apresentaram maiores prevalências de transtornos afetivos. No estudo OPCS, as pessoas com depressão maior apresentaram menor escolaridade e situação econômica inativa do que aquelas sem depressão ou outro transtorno mental.

Com relação à situação conjugal, a depressão parece ser mais freqüente entre pessoas divorciadas ou separadas, do que entre solteiros e casados. Viuvez recente está associada à alta ocorrência de depressão. Esses riscos parecem variar de acordo com o

Tabela 1 - Principais investigações epidemiológicas

\begin{tabular}{|c|c|c|c|c|c|c|}
\hline Estudo & Amostra (n) & Prevalência & Instrumento & $\begin{array}{l}\text { depressão } \\
\text { maior (\%) }\end{array}$ & Distimia (\%) & $\begin{array}{l}\text { Total transtornos } \\
\text { depressivos (\%) }\end{array}$ \\
\hline ECA & 19.182 & toda a vida & DIS & 4,9 & 3,2 & 6,3 \\
\hline NCS & 8.098 & 1 ano & CIDI & 10,3 & 2,5 & 12,8 \\
\hline $\begin{array}{l}\text { OPCS } \\
\text { Multicêntrico }\end{array}$ & 10.108 & 1 semana & CIS-R & 2,2 & $7,7^{*}$ & 10,0 \\
\hline Brasileiro** & 6.476 & toda a vida & $\begin{array}{l}\text { QMPA; } \\
\text { DSM-III }\end{array}$ & $\begin{array}{l}\text { Não } \\
\text { Disponível }\end{array}$ & $\begin{array}{l}\text { Não } \\
\text { Disponível }\end{array}$ & $\begin{array}{c}\text { 2,8 (Brasília) } \\
\text { 0,9 (São Paulo) } \\
\text { 10,2 (Porto Alegre }\end{array}$ \\
\hline
\end{tabular}

* No estudo conduzido no Reino Unido, o transtorno misto de ansiedade e depressão foi o transtorno neurótico mais prevalente. Não foi fornecida estimativa de prevalência para a distimia.

**No estudo Multicêntrico, os critérios diagnósticos da DSM-III foram aplicadas a uma sub-amostra de 836 pessoas e os resultados ponderados para a amostra total. Os resultados são relativos a 'transtornos depressivos'. 
sexo. Mulheres solteiras parecem ser menos suscetíveis à depressão do que casadas. Com os homens ocorre a situação oposta.

As pessoas que moram sozinhas parecem estar em maior risco para depressão: quanto maior o número de pessoas que moram no domicílio, menos a chance de apresentar depressão. A existência de suporte social e ausência de conflitos graves parecem ser agentes altamente protetores.

Eventos vitais: mudanças identificáveis no padrão de vida que representam uma ruptura do comportamento usual e afetam o bem estar do indivíduo. A maioria dos estudos epidemiológicos mostra uma associação entre eventos negativos e depressão maior. Existem problemas metodológicos relativos à forma como os eventos são estudados. Os eventos podem ter diferentes impactos de acordo com o período da vida, e parece que mais importante que o evento em si é a percepção do mesmo. No entanto, o acúmulo de eventos parece ser um fator que predispõe a pessoa a episódios depressivos. Um estudo conduzido em Pelotas (RS) ${ }^{12}$ mostrou que as pessoas que sofreram mais de dois eventos vitais têm duas vezes e meia mais chance de apresentar transtornos psiquiátricos menores do que aquelas que não tiveram nenhum evento no último ano. Os eventos estudados foram separação conjugal, perda por morte de ente querido, perda de emprego, acidente com lesão corporal, assalto, migração e presença de familiar com doença crônica no domicílio, ocorridos no último ano. A fração atribuível na população relacionada aos eventos vitais encontrada neste estudo foi $24 \%$ menor do que as relatadas por outros investigadores, que variam entre $30 \%$ e $50 \%$. No entanto, o estudo brasileiro foi conduzido na população geral, em que a ocorrência de eventos é baixa, quando comparadas com amostras clínicas, explicando a diferença com relação a outros estudos, conduzidos na sua maioria em populações clínicas ou selecionadas.

Estresse crônico: condições que a longo prazo trazem dificuldades, tais como dificuldades financeiras e nas relações interpessoais, e ameaças permanentes à segurança do indivíduo (morar em uma vizinhança perigosa). No estudo conduzido em Pelotas, ${ }^{12}$ a fração etiológica encontrada para educação foi superior a $50 \%$, o que sugere que se fosse proporcionado a toda a população educação superior poder-se-ia reduzir a freqüência de transtornos psiquiátricos menores à metade. Alguns autores como Wheaton ${ }^{13}$ consideram que fatores como baixa escolaridade podem causar estresse a longo prazo, particularmente para os analfabetos.

Suporte social: fatores sócioambientais podem modificar o efeito de estressores sobre as pessoas. Considera-se suporte social uma série de fatores protetores significantes e apropriados, que o ambiente é capaz de prover aos indivíduos, habilitando-os a lidar com estressores ambientais. $\mathrm{O}$ suporte social pode ser concebido a partir de quatro componentes: rede social, interação social, suporte social percebido, e suporte instrumental. Estudos epidemiológicos mostram que fatores como falta de esposo(a), isolamento social, e falta de uma pessoa confidente associam-se a maior ocorrência de depressão maior.

Residência: alguns estudos como o ECA sugerem que a ocorrência de depressão maior é cerca de duas vezes maior em comunidades urbanas, o que poderia estar relacionado à maior integração e menor estresse nesses locais. Essas diferenças se- riam maiores entre os jovens (abaixo de 45 anos de idade). Nesse estudo, tais diferenças permaneceram mesmo após controle de fatores de confusão, tais como raça, situação conjugal, idade e situação sócioeconômica. Na pesquisa OPCS, moradores de zonas urbanas apresentaram maiores prevalência de depressão maior. De forma consistente, o desemprego associa-se à maior ocorrência de depressão.

É importante salientar que os fatores acima citados formam uma rede causal, sendo que cada fator não apenas afeta diretamente o indivíduo, como também interage com os demais. Por isso, modelos matemáticos de fatores causais, como regressões lineares e logísticas, são úteis para determinar a importância relativa de cada um desses fatores. No estudo ECA, o resultado de análise de regressão logística mostrou que a depressão é mais freqüente entre jovens, mulheres e pessoas que moram na zona urbana, mesmo quando outros fatores sóciodemográficos são considerados.

\section{Prognóstico}

Um estudo de seguimento foi aninhado ao ECA na Carolina do Norte, objetivando acompanhar indivíduos com depressão maior ou distimia, e com sintomas de depressão menor, durante um ano. Os resultados indicam que pessoas com depressão maior ou distimia apresentam cerca de cinco vezes mais alguma forma de incapacidade do que indivíduos assintomáticos. Já aqueles com sintomas de depressão menor apresentam um excesso de $50 \%$ de risco. Outros estudos mostram que as pessoas deprimidas apresentam prejuízo no funcionamento social e físico.

\section{Uso de serviços de saúde}

As pessoas com transtornos depressivos consultam e se hospitalizam mais do que as assintomáticas, como mostram pesquisas internacionais e brasileiras. As mulheres utilizam mais serviços de saúde em geral do que os homens. No estudo OPCS, pessoas com transtornos mistos de ansiedade e depressão e depressão maior consultaram mais generalistas e se internaram mais freqüentemente que aquelas sem transtornos mentais. De acordo com o ECA, as pessoas mais idosas costumam procurar mais serviços de atenção primária à saúde. Os mais jovens procuram serviços especializados.

No estudo conduzido em Pelotas, a ocorrência de transtornos psiquiátricos menores associou-se positivamente a um excesso de consultas médicas nos três meses anteriores à entrevista e a hospitalizações por diversas razões, no último ano.

\section{Impacto social}

O impacto social da depressão inclui tanto a incapacidade individual como o fardo familiar associado à doença. Sintomas depressivos e depressão maior são problemas comuns e importantes em termos de saúde pública. Os custos da assistência médica, tempo de trabalho perdido, e a diminuição da qualidade de vida associam-se de forma clara e consistente com transtornos depressivos. Apesar de serem transtornos crônicos, os transtornos depressivos são condições tratáveis, e as opções terapêuticas têm crescido enormemente nos últimos 25 anos. Além disso, mesmo os sintomas menos severos de depressão são rele- 
vantes, na medida em que sua presença é associada ao desenvolvimento de outros transtornos psiquiátricos mais severos.

Um estudo conduzido no Reino Unido, em 1990, comparou os custos do tratamento farmacológico da depressão com imipramina e paroxetina, relacionando-os com os custos diretos da doença. ${ }^{14}$ Foi estimado que o custo total para o Reino Unido dos transtornos depressivos seria em torno de 222 milhões de libras. Os resultados mostraram ainda que os custos por paciente foram similares para ambas as drogas e que, quando se considera apenas os pacientes que obtiveram sucesso terapêutico, os custos são menores para paroxetina. Ainda que os tratamentos disponíveis possam representar um alto custo para os governos, parece que os altos custos diretos e indiretos da depressão excedem os valores associados com tratamentos eficazes, como o farmacológico.

\section{Conclusões}

Os transtornos depressivos são altamente prevalentes, tendem a afetar adultos jovens e apresentam um curso episódico ou crônico. São geralmente mais comuns em mulheres e em pessoas com menores rendas e níveis de escolaridade, e estão associados a uma alta utilização de serviços de saúde. As categorias diagnósticas distintas, como a depressão maior e distimia, apresentam distribuição semelhante e partilham os mesmos fatores de risco. Os achados de estudos populacionais sugerem que maior atenção deve ser dada a transtornos de severidade e duração menor, já que essas condições também se associam à incapacidade e prejuízo social, além de constituírem-se em condições de risco para o desenvolvimento de transtornos depressivos mais severos. Além do mais, sua freqüência na população é maior.

Como foi visto com relação aos fatores de risco e prognósticos, ainda são necessários estudos epidemiológicos adicionais. Apesar dos progressos em epidemiologia psiquiátrica, ainda são muitas as questões sem resposta com relação à depressão.

A alta taxa de utilização de serviços de saúde pelas pessoas sofrendo de depressão sugere que uma atenção especial deve ser dispensada no planejamento da política de saúde para essas condições. Pesquisas abordando a questão da depressão como um processo contínuo, baseadas na população geral, podem esclarecer mais a respeito dos mecanismos etiológicos e protetores da depressão, e, conseqüentemente, orientar ações em saúde a fim de prevenir a morbidade e o sofrimento individual e familiar associado à depressão.

\section{Referências bibliográficas}

1. Robbins N, Regier DA. Psychiatric disorders in America: the epidemiologic Catchment Area study. Lybrary of Congress Cataloging-in-Publication Data. New York; 1991

2. Blazer D. Mood disorders: epidemiology. In: Kaplan HI, Sadock BJ, editors. Comprehensive Textbook of Psychiatry. 6th ed. Baltimore MD: Williams \& Wilkins; 1995.

3. American Psychiatric Association. Diagnostic and Statistical Manual of Mental Disorders. 4th ed. Washington (DC): American Psychiatric Association; 1994.

4. Organização Mundial da Saúde. Classificação de Transtornos Mentais e de comportamento da CID-10. Porto Alegre: Artes Médicas; 1993

5. Golbberg D, Huxley P. Common Mental Disorders. A bio-social model.

London: Tavistock/Routledge; 1992.

6. Kessler RC, McGonagle KA, Zhao S, et al. Lifetime and 12-month prevalence of DSM-III-R psychiatric disorders in the United States. Archives of General Psychiatry 1994;51:8-19.

7. Jenkins R, Meltzer H. The National Survey of Psychiatric Morbidity in Great Britain. Soc Psychiatry Psychiatr Epidemiol 1995;30:1-4.

8. Mason P, Wilkonson G. The prevalence of psychiatric morbidity. OPCS survey of psychiatric morbidity in Great Britain. Br J Psychiatry 1996;168:1-3.

9. Almeida-Filho N, Mari JJ, Coutinho E, Franca JF, Fernandes J, Andreoli SB, et al. Brazilian multicentric study of psychiatric morbidity. Methodological features and prevalence estimates. Br J Psychiatry 1997;171:524-9.

10. Hagnell O, Lanke J, Rorsman B, Ojesjo L. Are we entering an age of melancholy? Depressive illness in a prospective epidemiological study over 25 years: The Lundby Study, Sweden. Psychol Med 1982;12:279-89.

11. Mari JJ, Almeida-Filho N, Coutinho E, Andreoli SB, Miranda CT, Streiner D. The epidemiology of psychotropic use in the city of São Paulo. Psychological Medicine 1993;23:467-74.

12. Lima MS, Béria JU, Tomasi E, Conceição AT, Mari JJ. Stressful life events and minor psychiatric disorders: an estimate of the population attributable fraction in a Brazilian community-based study. Int J Psychiatry Med 1996;26:213-24.

13. Wheaton B. The sociogenesis of psychological disorder: an attributional theory. Journal of Health and Social Behavior 1980;21:100-24.

14. Jonsson B, Bebbington PE. What price depression? The cost of depression and the cost-effectiveness of pharmacological treatment. Br J Psychiatry 1994;164:665-73.

Correspondência Maurício Silva de Lima

R. 15 de Novembro, 1089 - Pelotas, RS. CEP: 96015-000 